Revue d'histoire de l'Amérique française

REVUE D.HISTOIRE DE L'AMÉRIQUE FRANÇAISE

\title{
Praticiennes et patientes : Les femmes et la santé dans l'historiographie québécoise
}

\section{Denyse Baillargeon}

Volume 53, numéro 1, été 1999

Médecine, santé et sociétés

URI : https://id.erudit.org/iderudit/005319ar

DOI : https://doi.org/10.7202/005319ar

Aller au sommaire du numéro

Éditeur(s)

Institut d'histoire de l'Amérique française

ISSN

0035-2357 (imprimé)

1492-1383 (numérique)

Découvrir la revue

Citer cet article

Baillargeon, D. (1999). Praticiennes et patientes : Les femmes et la santé dans l'historiographie québécoise. Revue d'histoire de l'Amérique française, 53(1),

47-59. https://doi.org/10.7202/005319ar
Résumé de l'article

Cet article vise à tracer un bilan des recherches historiques québécoises, mais aussi canadiennes-anglaises, qui se sont intéressées au thème des femmes et de la santé. Deux perspectives y sont explorées : celle des praticiennes, essentiellement les infirmières, et celle des patientes, en particulier les mères. Centré autour de la question de l'autonomie des femmes vis-à-vis des médecins et de la dimension " genrée " des activités soignantes, ce bilan veut faire ressortir l'importance de prendre les femmes en compte dans toute étude portant sur l'univers de la santé, car elles ont certainement été les plus nombreuses à dispenser des soins et à les recevoir. 


\title{
PRATICIENNES ET PATIENTES \\ LES FEMMES ET LA SANTÉ DANS L'HISTORIOGRAPHIE QUÉBÉCOISE ${ }^{1}$
}

\author{
DENYSE BAILLARGEON \\ Département d'histoire \\ Université de Montréal
}

\section{RÉSUMÉ}

Cet article vise à tracer un bilan des recherches historiques québécoises, mais aussi canadiennes-anglaises, qui se sont intéressées au thème des femmes et de la santé. Deux perspectives y sont explorées : celle des praticiennes, essentiellement les infirmières, et celle des patientes, en particulier les mères. Centré autour de la question de l'autonomie des femmes vis-à-vis des médecins et de la dimension « genrée » des activités soignantes, ce bilan veut faire ressortir l'importance de prendre les femmes en compte dans toute étude portant sur l'univers de la santé, car elles ont certainement été les plus nombreuses à dispenser des soins et à les recevoir.

\section{ABSTRACT}

This article reviews the recent literature coming out of Quebec and English-speaking Canada on the topic of women and health. Two perspectives are explored here: that of practitioners, mainly nurses, and that of patients, particularly mothers. Centered on the issue of women's autonomy relative to doctors and also on the gendered aspects of healing, this review tries to convey the importance of taking women into account in any study of health and healing, given with their preponderant numbers as both givers and receivers of health care.

Depuis les débuts de l'histoire des femmes, la question des rapports entre les femmes et la santé a fait l'objet de très nombreuses recherches. D'une part, parce que le champ de la santé a été l'un des premiers, avec l'enseignement, à offrir des possibilités de « carrières féminines », dans les soins infirmiers notamment, et, d' autre part, parce que la capacité de reproduction des femmes a été, surtout depuis le $\mathrm{XIX}^{\mathrm{e}}$ siècle, un enjeu social et politique de première importance, ce qui a conduit les élites masculines, et en particulier le corps médical, à vouloir en régir les moindres dimensions.

1. Je remercie le Fonds pour la formation de chercheurs et l'aide à la recherche (FCAR) pour son soutien financier. Mes sincères remerciements s'adressent également à Nathalie Pilon qui a contribué à la préparation de ce bilan par des recherches bibliographiques et la préparation de synthèses. 
Sans prétendre épuiser le sujet, cet article se propose de tracer un bilan des recherches historiques qui se sont intéressées au groupe numériquement le plus important de praticiennes de la santé, à savoir les infirmières, et au processus de médicalisation de la maternité tout au long du $\mathrm{XX}^{\mathrm{e}}$ siècle. Essentiellement, il s'agira de retracer les interrogations qui ont guidé les historiennes du Québec, tout en tenant compte de ce qui s'est écrit ailleurs au Canada; de faire état des débats les plus importants que ces travaux ont suscités; et, enfin, de proposer quelques pistes de recherches. La question de l'autonomie des femmes vis-à-vis des médecins, qu'elles soient praticiennes ou patientes, constituera l'un des fils conducteurs de ce bilan, car elle a été au centre d'un grand nombre des études recensées; la dimension "genrée » des activités soignantes, que ce soit à l'intérieur ou à l'extérieur de la famille, représentera l'autre thème unificateur. Ce faisant, ce bilan espère faire ressortir l'importance de prendre les femmes en compte dans toute étude portant sur l'univers de la santé, car elles ont certainement été les plus nombreuses à dispenser des soins et à les recevoir, tout comme elles se sont le plus souvent retrouvées au centre des campagnes de santé publique et ont grandement participé à la diffusion du message hygiéniste, un des phénomènes les plus marquants de l'histoire de la médecine au $\mathrm{XX}^{\mathrm{e}}$ siècle.

\section{PRATICIENNES...}

De manière pratiquement unanime, les historiennes ont constaté que, de toutes les occupations féminines, le nursing est probablement celle qui a été la plus étudiée, d'abord par les praticiennes elles-mêmes (quand ce n'était pas par des médecins), puis à partir des années 1970, par une nouvelle génération d'historiennes féministes ${ }^{2}$. Au passage, la vision du nursing s'en est trouvée profondément modifiée : alors que les premiers travaux avaient mis en valeur le rôle progressiste joué par des pionnières comme Florence Nightingale dans la réforme du nursing à compter du $\mathrm{XIX}^{\mathrm{e}}$ siècle et qu'ils avaient insisté sur les luttes quasi héroïques des infirmières pour obtenir une formation médicale plus solide et se tailler une place au sein du monde des professions ${ }^{3}$, les études plus récentes donnent

2. Ce fait est souligné, entre autres, par Johanne Daigle, Devenir infirmière : le système d'apprentissage et la formation professionnelle à l'Hôtel-Dieu de Montréal, 1920-1970, thèse de doctorat (histoire), Université du Québec à Montréal, 1990, 20 et Veronica Strong-Boag, «Making a Difference : The History of Canada's Nurses », Canadian Bulletin of Medical History/Bulletin canadien d'histoire de la médecine (CBMH/BCHM), 8, 2 (1991) : 232.

3. Pour un exemple québécois de ce genre d'études, voir Édouard Desjardins et al., Histoire de la profession infirmière au Québec (Montréal, Association des infirmières et infirmiers de la province du Québec, 1970). 
à voir un univers féminin beaucoup plus complexe et ambigu. De fait, à partir du moment où l'histoire du nursing a commencé à se dissocier du champ de l'histoire plus traditionnelle de la médecine pour s'identifier à l'histoire des femmes, de l'éducation et à l'histoire sociale de la médecine, la question des rapports de pouvoir entre médecins et infirmières et la dimension genrée de la profession sont apparues comme des données incontournables.

Les premières études «féministes » du nursing se sont pourtant d'abord attardées aux mêmes thèmes que leurs prédécesseurs, à savoir les luttes professionnelles. Mais le mouvement de professionnalisation du nursing, qui s'est traduit par la création des premières écoles d'infirmières au tournant du siècle et qui s'est poursuivi dans l'arène législative, afin de restreindre le droit de pratique à celles qui avaient été ainsi formées, a suscité de nouvelles interrogations et bien des remises en question. Un des débats qui a sans doute le plus marqué cette nouvelle production historiographique a tourné autour d'une question en particulier : peut-on, à la lumière du modèle masculin de professionnalisation, dont la médecine semble représenter le prototype, considérer le nursing comme une profession? Les historiennes féministes ont plutôt souligné les paradoxes qui restent attachés à la profession d'infirmière, jusqu'à remettre en cause l'idée même qu'elle en soit une.

Plusieurs ont en effet souligné le faible statut social attaché au nursing, les conditions de travail difficiles, la rémunération dérisoire en regard des qualifications requises, le fait que la formation soit axée sur la pratique plutôt que sur la théorie et que les infirmières n'aient pas obtenu une pleine autonomie d'exercice ni une autorégulation complète qui caractérisent les autres professions comme la profession médicale ${ }^{4}$. En fait, la plupart des historiennes insistent pour dire que le corps médical, en s'arrogeant le droit exclusif de diagnostiquer et de prescrire, s'est assuré de dominer le champ de la santé, ce qui lui a permis d'exclure plusieurs catégories de soignants et d'exercer sa domination sur les autres praticiens, en particulier les infirmières ${ }^{5}$. Dans cette perspective, le nursing est considéré par certains comme une semi-profession, une profession de deuxième classe ou une profession dépendante. Compte tenu de

4. À ce sujet, voir les bilans historiographiques de J. Daigle, op. cit., 20-63; Kathryn McPherson et Meryn Stuart, "Writing Nursing History in Canada : Issues and Approaches », CBMH/BCHM, 11,1 (1994) : 3-22, et Kathryn McPherson, Bedside Matters. The Transformation of Canadian Nursing, 1900-1990 (Toronto, Oxford University Press, 1996), 1-18.

5. Au sujet de la montée de la profession médicale au Québec, voir Jacques Bernier, La médecine au Québec : naissance et évolution d'une profession (Québec, Les Presses de l'Université Laval, 1989, et Nadia Fahmy-Eid et al., Femmes, santé et professions. Histoire des diététistes et des physiothérapeutes au Québec et en Ontario 1930-1980 (Montréal, Fides, 1997), en particulier le chapitre 6, 143-166. 
la centralisation des soins infirmiers dans les hôpitaux, de la standardisation et de la rationalisation des procès de travail tout au long du $\mathrm{XX}^{\mathrm{e}}$ siècle, d'autres en sont arrivés à la conclusion que le concept de prolétarisation convenait bien davantage que celui de professionnalisation pour décrire les transformations que le nursing a connues, surtout après la Deuxième Guerre mondiale ${ }^{6}$.

Certaines historiennes ont aussi souligné que le processus de professionnalisation n'est pas attribuable uniquement à la détermination dont auraient fait preuve les infirmières, comme le suggéraient les études pionnières à leur sujet. Ce mouvement proviendrait également de la volonté des administrations hospitalières de se moderniser, afin de faire de l'hôpital un lieu de guérison plutôt qu'un mouroir, et du désir des médecins, du moins d'une partie du corps médical, de travailler en compagnie d'assistantes mieux formées. Selon Yolande Cohen et Louise Bienvenue, par exemple, l'enjeu de la professionnalisation des infirmières pourrait s'exprimer en ces termes : subordonner le travail infirmier à celui des médecins, tout en s'assurant de sa qualité. C'est pourquoi, selon elles, les médecins appuieront les demandes des infirmières pour avoir accès à une meilleure formation, mais s'opposeront, du moins chez les franco-catholiques, à leur volonté de régir l'accès à la profession ${ }^{7}$. Dans son étude sur le nursing au Canada, Kathryn McPherson va encore plus loin en affirmant que l'ascension de la profession médicale au XIX ${ }^{\mathrm{e}}$ siècle s'est carrément appuyée sur la création d'un groupe d'auxiliaires qualifiées, essentielles pour exécuter les traitements médicaux scientifiques recommandés par les médecins ${ }^{8}$.

La subordination des infirmières aux médecins ne peut évidemment se comprendre sans référence aux rapports de genre et à la construction sociale d'une identité féminine axée sur le care ou le caring. Comme le notent à peu près tous les auteurs, les leaders du nursing n'ont à peu près jamais contesté l'association entre nursing et capacité « naturelle » des femmes à soigner et à se dévouer pour les autres, et elles n'ont pas non plus cherché à s'opposer à la subordination du travail infirmier à celui des médecins ${ }^{9}$. De la sorte, il devenait sans doute plus facile de faire accepter

6. Kathryn McPherson fait une excellente revue de ces différentes analyses dans op. cit., 7-15.

7. Yolande Cohen et Louise Bienvenue, «Émergence de l'identité professionnelle chez les infirmières québécoises, 1890-1927 », CBMH/BCHM, 11,1 (1994) : 119-151.

8. K. McPherson, op. cit., 13.

9. Barbara Melosh, "The Physician's Hand»: Work, Culture and Conflict in American Nursing (Philadelphie, Temple University Press, 1982); Louise-Hélène Trottier, Évolution de la profession infirmière au Québec de 1920 à 1980, mémoire de maîtrise (sociologie), Université de Montréal, 1982, citée dans J. Daigle, op. cit., 30. 
la présence des infirmières dans l'univers de la santé et de leur donner accès à un savoir scientifique, source de prestige et de légitimité. Mais du même coup, celles-ci se sont enferrées dans une logique qui a largement contribué à maintenir cette occupation dans une position d'infériorité ${ }^{10}$.

Plus récemment, certaines historiennes ont cependant souligné que, comparé à bien d'autres occupations féminines, le nursing présentait plusieurs avantages pour les femmes, en particulier la possibilité de travailler dans un environnement non industriel, d'assurer leur indépendance économique grâce à des salaires décents et de jouir d'un statut enviable dans leur communauté. Plutôt que de comparer leur prestige, leurs conditions de travail et leur autonomie professionnelle avec les médecins, elles suggèrent de situer le nursing sur l'échelle des occupations féminines de manière à rendre compte de ce qu'il a véritablement signifié pour les femmes ${ }^{11}$. Au portrait plutôt sombre de la profession qui se dégage de la stricte comparaison médecin-infirmière se substitue donc, dans certains travaux plus récents, une image plus positive du nursing tant sur le plan des gains professionnels que sur celui du statut social pour les femmes. Kathryn McPherson suggère pour sa part de considérer le nursing d'abord et avant tout comme un travail et d'en analyser les différentes composantes, une dimension de l'histoire des infirmières qui a trop souvent été occultée selon elle en raison justement de la place qu'a prise la question de leur statut professionnel ${ }^{12}$. Déjà au début des années 1980, Marie Lavigne et Jennifer Stoddart en étaient arrivées au même constat ${ }^{13}$. D'ailleurs, comme l'ont écrit Yolande Cohen et Michèle Dagenais, les infirmières n'ont pas nécessairement défendu l'option de la professionnalisation avec la même vigueur. Ainsi, à Montréal, les infirmières anglophones se sont montrées plus désireuses que leurs consœurs francophones d'améliorer la formation et de régir l'accès à la profession par l'entremise d'une association qu'elles voulaient autonome ${ }^{14}$. Faut-il voir un lien entre, d'une part, le peu d'intérêt manifesté

10. Dianne Dodd et Deborah Gorham, «Introduction », dans Dianne Dodd et Deborah Gorham, dir., Caring and Curing : Historical Perspectives on Women and Healing in Canada (Ottawa, Presses de 1'Université d'Ottawa, 1994), 1-17.

11. Voir en particulier Mary Kinnear, In Subordination : Professional Women, 1870-1970 (Montréal, McGill-Queen's University Press, 1995); Y. Cohen et L. Bienvenue, op. cit. Le statut professionnel des infirmières pourrait ainsi se comparer à celui des employées de bureau étudiées par Michèle Dagenais dans « Itinéraires professionnels masculins et féminins en milieu bancaire : le cas de la banque d'Hochelaga, 1900-1929 », Labour/Le Travail, 24 (automne 1989) : 445-468.

12. K. McPherson, op. cit., 2.

13. Marie Lavigne et Jennifer Stoddart, « Ouvrières et travailleuses montréalaises, 19001940 », dans Marie Lavigne et Yolande Pinard, dir., Travailleuses et féministes. Les femmes dans la société québécoise (Montréal, Boréal-Express, 1983), 113.

14. Yolande Cohen et Michèle Dagenais, «Le métier d'infirmière: savoirs féminins et reconnaissance professionnelle », Revue d'histoire de l'Amérique française (RHAF), 41, 2 (automne 1987) : 155-177. 
par les infirmières canadiennes-françaises envers l'Association des gardesmalades enregistrées de la province de Québec (AGMEPQ) et leurs réticences, sinon leur opposition, à l'enregistrement et, d'autre part, le fait qu'elles se soient syndiquées plus tôt qu' au Canada anglais, comme le révèle l'étude pionnière de Johanne Daigle sur l'Alliance des infirmières de Montréal ${ }^{15}$ ? Les travaux les plus récents tendent à montrer que l'idéal professionnel a d'abord été défendu par l'élite du nursing, avant de devenir un enjeu pour les infirmières de la base ${ }^{16}$ : le fait que l'élite des infirmières franco-catholiques, c'est-à-dire celles qui occupaient les postes de direction dans les hôpitaux, ait été presque toujours composée de religieuses qui n'avaient guère d'avantages, pécuniaires ou autres, à tirer de la professionnalisation, aurait-il fait en sorte que les laïques qui travaillaient sous leurs ordres auraient été moins en contact avec cet idéal professionnel et l'auraient donc défendu avec moins de conviction? Il est vrai que les religieuses ont contribué à la professionnalisation du nursing par les écoles d'infirmières qu'elles ont dirigées, un fait que démontrent les travaux de Johanne Daigle sur l'école des infirmières des Hospitalières de SaintJoseph de l'Hôtel-Dieu de Montréal, de Yolande Cohen sur les Sœurs de la Charité chargées des soins à l'hôpital Notre-Dame ou de François Rousseau sur les Augustines de l'Hôtel-Dieu de Québec ${ }^{17}$. Mais les médecins semblent avoir joué un rôle important dans ce processus et il est certain que la gestion de la majorité des hôpitaux québécois, ou de leurs soins infirmiers, par des communautés religieuses sous-financées par l'État, a fait en sorte de maintenir les conditions de travail et les salaires à la baisse. Tout en rendant cette profession «féminine » moins attirante pour l'ensemble des jeunes filles ${ }^{18}$, l'administration des hôpitaux par des religieuses a probablement milité en faveur d'une stratégie de syndicalisation.

15. Johanne Daigle montre que les infirmières québécoises se syndiquent dès les années 1940, alors qu'au Canada anglais, il faut attendre les années 1960 pour voir ce phénomène prendre un réel essor selon K. McPherson. J. Daigle, «L'éveil syndical des "religieuses laïques" : l'émergence et l'évolution de l'Alliance des infirmières de Montréal, 1946-1966 », dans Nadia Fahmy-Eid et Micheline Dumont, Maîtresses de maison, maîtresses d'écoles (Montréal, Boréal-Express, 1983), 115-138; K. McPherson, op. cit., 219-236.

16. V. Strong-Boag, loc. cit., 237.

17. J. Daigle, op. cit.; id., « Devenir infirmière — les modalités d'expression d'une culture soignante au XX ${ }^{\mathrm{e}}$ siècle », Recherches féministes, 4,1 (1991) : 67-86; Yolande Cohen, « La contribution des Sours de la charité à la modernisation de l'hôpital Notre-Dame, 1880-1940 », Canadian Historical Review (CHR), 77,2 (juin 1996) : 185-220; et François Rousseau, La Croix et le scalpel. Histoire des Augustines et de l'Hôtel-Dieu de Québec, II : 1892-1989 (Sillery, Septentrion, 1994).

18. Au sujet de l'impact de la présence massive des religieuses dans les professions dites féminines, voir Danièle Juteau, «Les religieuses au Québec : leur influence sur la vie professionnelle des Québécoises, 1909-1954 », Atlantis, 5, 2 (printemps 1980) : 22-33. 
Tout comme on a commencé à rejeter la dichotomie médecininfirmière en ce qui concerne l'appréciation du statut professionnel de ces dernières, on a aussi jeté un regard plus critique sur l'opposition entre le care et le cure à laquelle on s'est longtemps référé pour expliquer la subordination des infirmières aux médecins. Certes, comme l'a bien montré Johanne Daigle, la formation des infirmières au sein d'écoles affiliées aux hôpitaux a permis de développer une «culture soignante » proprement féminine, fondée sur l'acquisition d'un ensemble intégré de savoirs, de valeurs et de pratiques déterminés en fonction de l'appartenance de sexe et de l'identification des femmes aux activités soignantes. Comme Daigle le note cependant, ce modèle associé au caring pouvait difficilement se perpétuer sans modifications importantes, dans un contexte où les associations professionnelles cherchaient à augmenter la composante théorique de la formation des infirmières et où les avancées technologiques et scientifiques transformaient leurs pratiques et leurs rapports aux patients ${ }^{19}$. À ce propos, Kathryn McPherson et Meryn Stuart vont même jusqu'à dire que la médecine scientifique a constitué une force définissante à l'intérieur du nursing car, dès la fin du XIX ${ }^{\mathrm{e}}$ siècle, les associations d'infirmières, qui cherchaient à se faire accepter dans le monde des professionnels de la santé, ont embrassé les explications scientifiques de la maladie et de la thérapie ${ }^{20}$. Pour McPherson, la science, tant en ce qui a trait à la médecine scientifique qu'à la rationalisation du travail, se trouve au cœur même des tâches accomplies par les infirmières : intégrés à leur identité occupationnelle, ces savoirs scientifiques leur permettaient de se distinguer des autres soignantes sans formation, en particulier des mères de famille ${ }^{21}$.

En fait, tout au long du $\mathrm{XX}^{\mathrm{e}}$ siècle, les frontières entre le care et le cure ont été en constante mutation, car les médecins ont eu de plus en plus tendance à déléguer certains actes médicaux aux infirmières, qui ellesmêmes se déchargeaient de certaines de leurs responsabilités au profit de diverses catégories d'aides et de techniciennes dont elles supervisaient le travail. Ce jeu de dominos n'a d'ailleurs pas été sans susciter de nombreuses luttes inter et même intraprofessionnelles, comme en témoignent les recherches menées par Nadia Fahmy-Eid et son équipe au sujet des diététistes, des physiothérapeutes et des techniciennes médicales ${ }^{22}$. Si les infirmières sont hiérarchiquement subordonnées aux médecins, cette

19. J. Daigle, « Devenir infirmière », loc. cit.

20. K. McPherson et M. Stuart, loc. cit., 7-9.

21. K. McPherson, op. cit., 113.

22. N. Fahmy-Eid et al., op. cit., et Lucie Piché et N. Fahmy-Eid, « À la recherche d'un statut professionnel dans le champ paramédical. Le cas de la diététique, de la physiothérapie et de la technologie médicale (1940-1973), RHAF, 45,3 (hiver 1992) : 375-401. 
étude permet de constater qu'elles occupent une position stratégique qui leur a permis de défendre efficacement leur territoire professionnel face à la compétition et de préserver, du moins jusqu'aux décennies les plus récentes, le prestige qui en découle.

Les luttes de pouvoir que les professionnelles de la santé, en particulier les infirmières, ont dû livrer à d'autres femmes pour consolider leurs assises est d'ailleurs un thème qui est plus fréquemment exploré depuis quelques années. Ainsi, dans son étude sur le bénévolat à l'hôpital SainteJustine, Aline Charles a montré comment le monde des bénévoles a été bousculé par le mouvement de professionnalisation des soins et la construction de champs de pratiques exclusifs ${ }^{23}$. J'ai pu constater le même processus de marginalisation des bénévoles à l'Assistance maternelle au profit des infirmières ${ }^{24}$. Pour mieux s'affirmer comme des professionnelles à part entière, les infirmières se sont souvent comportées comme les «alliées objectives » des médecins, disent certaines historiennes, en dévalorisant les savoirs traditionnels des mères de famille et en s'opposant à la pratique des sages-femmes ou, plus récemment, aux groupes féministes qui revendiquaient, pour les femmes, la maîtrise sur leur propre corps et une « humanisation» de la naissance. McPherson constate pour sa part que, pendant longtemps, le nursing a été réservé aux femmes blanches d'origine anglo-saxonne, soulignant par là que la « race », tout autant que le genre a contribué à façonner l'identité des infirmières ${ }^{25}$.

Enfin, depuis environ une décennie, l'intérêt des historiennes s'est tourné vers d'autres catégories d'infirmières, en particulier celles œuvrant dans la santé publique. D'une certaine manière, l'infirmière visiteuse ou hygiéniste est apparue comme une élue parmi les élues, en raison du statut particulièrement prestigieux dont elle bénéficiait dans sa communauté et des salaires élevés (à l'échelle féminine bien sûr) qu'elle pouvait obtenir. Meryn Stuart, entre autres, a cependant fait remarquer que, si les autorités en santé publique ont choisi de recourir à des infirmières pour faire le travail d'éducation des masses, c'est, d'une part, parce qu'elles coûtaient moins cher et, d'autre part, parce qu'elles pouvaient, davantage que les hommes, s'attirer la confiance de la clientèle visée, essentiellement féminine, et qu'elles étaient familières avec l'environnement domestique où elles étaient

23. Aline Charles, Travail d'ombre et de lumière. Le bénévolat féminin à l'hôpital Sainte-Justine 1907-1960 (Québec, Institut québécois de recherche sur la culture, coll. « Edmond-de-Nevers », $\left.\mathrm{n}^{\circ} 9,1990\right)$.

24. Denyse Baillargeon, «L'Assistance maternelle de Montréal. Un exemple de marginalisation des bénévoles dans le domaine des soins aux accouchées », Dynamis, numéro spécial, Mujeres y salud. Prácticas y saberes/Women and Health. Practices and Knowledges, 19 (1999) : 379-400.

25. K. McPherson, op. cit., 17. 
appelées à travailler ${ }^{26}$. Selon plusieurs historiennes, le fait que les infirmières exerçaient leur métier en dehors des hôpitaux a conduit le corps médical à vouloir régir encore plus strictement leurs pratiques. L'histoire de la clinique ouverte par la compagnie d'assurance-vie La Métropolitaine à Thetford Mines montre que les infirmières hygiénistes ont souvent été en butte à l'opposition farouche des médecins généralistes qui craignaient de perdre leur clientèle et leurs revenus, ce qui s'est parfois traduit par des menaces de poursuites judiciaires ${ }^{27}$. Cet épisode témoigne aussi du fait que les infirmières hygiénistes étaient souvent plus au fait que les généralistes des derniers développements en matière de santé publique. Comme le note Meryn Stuart, ces infirmières se sont souvent trouvées dans des situations impossibles. À certaines occasions, l'absence de médecin pouvait les obliger à prendre des décisions qui impliquaient qu'elles avaient bel et bien posé un diagnostic, ce qui en théorie leur était totalement interdit; à d'autres moments, elles faisaient face à des médecins totalement ignorants en matière de médecine préventive et elles devaient alors faire leur éducation, tout en maintenant une attitude de soumission respectueuse ${ }^{28}$. Étudiant l'émergence de cette profession chez les Canadiennes françaises, Yolande Cohen et Michèle Gélinas constatent, pour leur part, que les infirmières hygiénistes ont été les premières à se laïciser, le modèle de la santé publique laissant moins de place à la sauvegarde des âmes qui faisait partie intégrante de la mission des religieuses-infirmières exerçant dans les hôpitaux ${ }^{29}$. Enfin, les travaux de Johanne Daigle et Nicole Rousseau montrent que la nécessité d'assurer un minimum de services médicaux dans les régions de colonisation a forcé l'État québécois à accorder de larges prérogatives aux infirmières de colonies, contrevenant ainsi aux normes professionnelles en vigueur. Plus que toute autre catégorie d'infirmières, les infirmières de colonie ont bénéficié d'une très large autonomie dans leur pratique, car elles étaient considérées comme des substituts des médecins. En contrepartie, elles ont eu à supporter des conditions de travail particulièrement dures : dispensaires inadéquats, manque de matériel et de médicaments, isolement, etc. En fait, on peut dire que n'eût été de ce contexte difficile qui rebutait les médecins, elles n'auraient pu se tailler cette niche exceptionnelle. D'ailleurs, lorsque

26. M. Stuart, « Let Not the People Perish for Lack of Knowledge » : Public Health Nursing and the Ontario Rural Child Welfare Project, 1916-1930, thèse de doctorat, Université de Pennsylvanie, 1987; et id., « Shifting Professional Boundaries : Gender Conflict in Public Health, 1920-1925 », dans D. Dodd et D. Gorham, dir., Caring and Curing, op. cit., 49-70.

27. Denyse Baillargeon, «Les rapports médecins-infirmières et l'implication de la Métropolitaine dans la lutte contre la mortalité infantile, 1909-1953 », CHR, 77,1 (mars 1996) : 53 et ss.

28. M. Stuart, dans D. Dodd et D. Gorham, dir., Caring and Curing, op. cit.

29. Yolande Cohen et Michèle Gélinas, « Les infirmières hygiénistes de la ville de Montréal : du service privé au service civique », Histoire sociale/Social History, 22,44 (novembre 1989) : 219-246. 
la situation de ces régions éloignées se sera quelque peu normalisée, les médecins reprendront leur droit et l'État verra à retirer les prérogatives consenties en raison de la nécessité et de l'urgence ${ }^{30}$.

\section{...ET PATIENTES}

Si on se tourne maintenant du côté des patientes, on constate que les travaux portant sur leurs rapports avec les médecins se sont attardés principalement à deux phénomènes : le discours médical sur les femmes en général et le processus de médicalisation de la grossesse et de l'accouchement qui a pris de plus en plus d'ampleur tout au long du $\mathrm{XX}^{\mathrm{e}}$ siècle. Des historiennes, comme Andrée Lévesque pour le Québec et Wendy Mitchinson pour le Canada anglais, ont bien montré comment le discours médical de la fin du $\mathrm{XIX}^{\mathrm{e}}$ et du début du $\mathrm{XX}^{\mathrm{e}}$ siècle était fortement influencé par les idéologies prévalant au sujet des rôles sociaux de sexes $^{31}$. S'appuyant sur la nouvelle autorité que leur conférait leur statut de professionnel, ces médecins ont contribué à cautionner «scientifiquement» les inégalités de genre et le supposé destin « naturel » des femmes, à savoir la maternité. Une fois le corps féminin posé comme « autre », en regard du corps masculin, déclaré intrinsèquement pathologique et réduit à sa fonction reproductrice, symbolisée par l'utérus, il ne restait qu'à affirmer que les femmes ne pouvaient se soustraire à leur biologie sans risquer les pires désordres physiologiques ou psychologiques. Ainsi, exercer le droit de vote, envisager des études supérieures, travailler en usine, recourir à la contraception ou refuser l'allaitement devenaient autant de comportements à dénoncer parce qu'ils allaient directement à l'encontre de la nature profonde des femmes et constituaient un risque non seulement pour elles-mêmes, mais aussi, et surtout, pour l'ordre social.

Si elles se sont déployées tous azimuts, les prescriptions médicales se sont faites particulièrement pressantes en ce qui concerne la grossesse et l'accouchement. Le désir des médecins d'encadrer les femmes « expectantes », comme on les appelait au début du siècle, et les parturientes provenait en bonne partie, disent plusieurs historiennes, de leur volonté de se bâtir une clientèle. L'élimination des sages-femmes du champ de

30. Johanne Daigle et Nicole Rousseau, «Le service médical aux colons. Gestation et implantation d'un service infirmier au Québec (1932-1943)», RHAF, 52,1 (été 1998) : 47-72.

31. Andrée Lévesque, « Mères ou malades : les Québécoises de l'entre-deux-guerres vues par les médecins », RHAF, 38,1 (été 1984) : 23-38; id., La norme et les déviantes. Des femmes au Québec pendant l'entre-deux-guerres (Montréal, Éditions du Remue-Ménage, 1989) ; Wendy Mitchinson, The Nature of their Bodies : Women and their Doctors in Victorian Canada (Toronto, University of Toronto Press, 1991). 
l'obstétrique, phénomène étudié par Hélène Laforce, représentait une première étape dans cette direction ${ }^{32}$ : il a ensuite fallu convaincre les femmes de la nécessité de consulter leur médecin tout au long de leurs grossesses et de suivre ses directives, avant de les amener à accoucher à l'hôpital. Plusieurs auteurs ont été particulièrement critiques face à ce processus. France Laurendeau, par exemple, notait que l'accouchement en milieu hospitalier équivalait à une véritable dépossession des femmes de leur maternité. Le transfert vers l'hôpital des accouchantes s'est en effet traduit, selon elle, par une perte de maîtrise des femmes sur leur environnement et par une multiplication des interventions médicales (anesthésie, épisiotomie), sans que l'on puisse attribuer à celles-ci la baisse de la mortalité maternelle, qui serait plutôt due à l'introduction des sulfamides puis des antibiotiques ${ }^{33}$.

D'une certaine manière, on peut dire que les premiers travaux qui se sont intéressés aux rapports entre les femmes et les médecins avaient tendance à négliger l'autre côté de l'équation, à savoir les femmes ellesmêmes. Comment recevaient-elles tous ces conseils et prescriptions? Dans quelle mesure s'y conformaient-elles? Qu'est-ce qui les amenait à les accepter ou à les rejeter? Autant de questions auxquelles les historiennes se sont ensuite attaquées et qui ont permis de relativiser l'image d'un corps médical tout-puissant. Ces recherches ont montré que, confrontées à la mortalité infantile et maternelle qui sévissait au début du siècle, les femmes étaient plutôt enclines à accorder le bénéfice du doute à leurs praticiens quand ils leur promettaient que la médecine moderne pouvait les sauver, elles et leurs bébés. Mais leur soumission aux diktats de la médecine n'était ni totale ni unanime, et les femmes avaient leur propres raisons de suivre les prescriptions médicales. Ainsi, Marie-Josée Blais a montré que les femmes des années 1940 et 1950 allaient accoucher à l'hôpital non pas uniquement pour y retrouver l'environnement sécuritaire tant vanté par les médecins, mais aussi parce que le séjour en milieu hospitalier représentait pour elles un temps d'arrêt, un moment dans leur vie où elles pouvaient se décharger de leurs responsabilités domestiques sans ressentir de la culpabilité ${ }^{34}$. De même, j' ai pu constater que, malgré les campagnes intensives faisant la promotion de l'allaitement maternel et du suivi prénatal, les femmes ont longtemps négligé ces directives. Faute de moyens financiers, les femmes de la classe ouvrière pouvaient certes difficilement fréquenter

32. Hélène Laforce, Histoire de la sage-femme dans la région de Québec (Québec, Institut québécois de recherche sur la culture, coll. «Edmond-de-Nevers », $n^{\circ} 4,1985$ ).

33. France Laurendeau, «La médicalisation de l'accouchement », Recherches sociographiques, 24,2 (mai-août 1983) : 203-234.

34. Marie-Josée Blais, Le transfert hospitalier de l'accouchement au Québec entre 1930 et 1960, mémoire de maîtrise (histoire) Université de Montréal, 1995. 
assidûment le cabinet d'un praticien, mais même les cliniques prénatales gratuites, comme celles mises sur pied par la Ville de Montréal ou l'Assistance maternelle, étaient dédaignées, ce qui démontre que les femmes n'étaient pas convaincues de la nécessité de se soumettre à des batteries de tests et d'examens. Quant aux cliniques de puériculture, je crois avoir montré que les mères y allaient autant pour socialiser avec d'autres mères et échanger des « trucs », ce que les médecins cherchaient justement à empêcher, que pour recevoir vaccins et conseils ${ }^{35}$.

Les recherches au sujet du développement de la santé publique ont par ailleurs révélé qu'une bonne partie des prescriptions médicales transmises aux mères, dans les Gouttes de lait par exemple, étaient en fait relayées par les infirmières qui, plus que les médecins, étaient en contact étroit avec les patientes. S'il faut parler d'une forme de pouvoir médical sur les mères, il faut donc admettre qu'une large part de cette domination était exercée par des femmes. Ce qui ne veut pas dire, par ailleurs, que l'autorité de l'infirmière était ressentie de manière négative par l'ensemble des femmes : les témoignages que j'ai recueillis auprès, il est vrai, de femmes qui étaient visiblement satisfaites des services reçus, montrent au contraire que l'infirmière était fort respectée, parfois même adulée. Ses conseils, même s'ils visaient à modifier des habitudes jugées mauvaises, étaient généralement bien reçus, surtout quand ils permettaient de gagner du temps et d'alléger le travail domestique. Dans ce cas, les mères de famille étaient même prêtes à accueillir favorablement la présence des infirmières jusque dans l'intimité de leur foyer : l'exemple du service des infirmières visiteuses de la Métropolitaine est ici probant ${ }^{36}$.

Bref, si le monde des praticiennes est de plus en plus réexaminé à la lumière des tensions et des divisions qui l'animent, il semble que l'on tente maintenant de s'attarder au point de vue des patientes, afin de relativiser l'idée que les femmes, en particulier les mères, n'ont fait que subir une domination médicale qu'elles n'auraient pas souhaitée. Le petit nombre de travaux qui se fondent sur les témoignages des femmes invite certainement à la prudence, mais d'ores et déjà, il est permis de conclure que les analyses inspirées de la thèse du « contrôle social » peuvent difficilement rendre compte de la complexité d'un phénomène comme la médicalisation de la maternité.

35. Denyse Baillargeon, « Fréquenter les Gouttes de lait. L'expérience des mères montréalaises, 1910-1965 », RHAF, 50,1 (été 1996) : 29-68.

36. Denyse Baillargeon, «Les infirmières de la Métropolitaine au service des Montréalaises », dans Les bâtisseuses de la cité (Montréal, Acfas, « Les cahiers scientifiques » n 79, 1993), 107-120. Actes du colloque « Les bâtisseuses de la cité », Section d'études féministes, congrès de l'Acfas, 1992. 


\section{CONCLUSION}

Comme le montre ce court bilan, les historiennes ont beaucoup plus étudié le monde des praticiennes que celui des patientes et, dans le cas de ces dernières, les travaux se sont surtout intéressés au phénomène de la médicalisation de la maternité. On ignore encore à peu près tout des rapports entre infirmières et patientes hospitalisées et entre patientes et bénévoles, qui ont pourtant été nombreuses à dispenser soins et réconfort dans les hôpitaux et les œuvres philanthropiques de nature médicale. À l'exception des travaux de Francine Saillant, peu d'études se sont attardées au rôle de soignante des mères ${ }^{37}$ et surtout à ses transformations sous l'effet de l'industrialisation et de l'urbanisation. La multiplication des médicaments, accessibles avec ou sans ordonnance, comme les avancées de la médecine qui ont simplifié certains actes médicaux, au point où la mère de famille a pu remplacer l'infirmière, laissent pourtant deviner que la médecine moderne n'a pas totalement dépossédé les femmes de ce rôle de soignante : il se serait plutôt modifié suivant des modalités et une chronologie qu'il reste à établir. Comme le faisaient remarquer McPherson et Stuart, les soins donnés par les infirmières de la Nouvelle-France se rapprochaient sans doute de ceux dispensés au foyer, tandis que les mères d'aujourd'hui disposent d'un arsenal maison plus sophistiqué que celui des infirmières du $\mathrm{XIX}^{\mathrm{e}}$ siècle pour prendre soin des leurs ${ }^{38}$. C'est dire que la frontière entre les soins professionnels et domestiques a été en constante redéfinition, une question qui a été peu explorée jusqu'à maintenant. Pourtant, elle est certainement d'actualité, compte tenu du désengagement de l'État dans le secteur de la santé qui a conduit à la désinstitutionnalisation massive des patients psychiatrisés il y a quelques années et, plus récemment, au virage ambulatoire qui compte sur les «aidants naturels », le plus souvent des « aidantes », pour être... opératoire.

37. Francine Saillant, «Savoirs et pratiques des femmes dans l'univers ethnomédical québécois », Canadian Folklore, 14,1 (1992) : 47-72, et F. Saillant et Hélène Laforce, « Médecine domestique et pratiques sociales entourant la reproduction chez les Québécoises », Canadian Folklore, 15,2 (1993) : 31-50.

38. K. McPherson et M. Stuart, op. cit., 6. 